\title{
A pseudoscalar decaying to photon pairs in the early LHC Run 2 data
}

\author{
Matthew Low, ${ }^{a}$ Andrea Tesi ${ }^{b}$ and Lian-Tao Wang ${ }^{c}$ \\ ${ }^{a}$ School of Natural Sciences, Institute for Advanced Study, \\ Princeton, NJ 08540, U.S.A. \\ ${ }^{b}$ Department of Physics, Enrico Fermi Institute, University of Chicago, \\ Chicago, IL 6063\%, U.S.A. \\ ${ }^{c}$ Department of Physics, Enrico Fermi Institute, and Kavli Institute for Cosmological Physics, \\ University of Chicago, Chicago, IL 60637, U.S.A. \\ E-mail: mattlow@ias.edu, atesi@uchicago.edu, liantaow@uchicago.edu
}

ABSTRACT: In this paper we explore the possibility of a pseudoscalar resonance to account for the $750 \mathrm{GeV}$ diphoton excess observed both at ATLAS and at CMS. We analyze the ingredients needed from the low energy perspective to obtain a sufficiently large diphoton rate to explain the signal while avoiding constraints from other channels. Additionally, we point out composite Higgs models in which one can naturally obtain a pseudoscalar at the $750 \mathrm{GeV}$ mass scale and we estimate the pseudoscalar couplings to standard model particles that one would have in such models. A generic feature of models that can explain the excess is the presence of new particles in addition to the $750 \mathrm{GeV}$ state. Finally, we note that due to the origin of the coupling of the resonance to photons, one expects to see comparable signals in the $Z \gamma, Z Z$, and $W W$ channels.

Keywords: Beyond Standard Model, Technicolor and Composite Models

ARXIV EPRINT: 1512.05328 


\section{Contents}

1 Motivation 1

2 Signals and constraints from the LHC 3

3 The interactions of a pseudoscalar 4

4 The mass scale of a pseudoscalar $\quad 11$

5 Alternative explanations $\quad \mathbf{1 5}$

$\begin{array}{lll}5.1 & \text { Scalar resonance } & 16\end{array}$

$\begin{array}{lll}5.2 & \text { Spin-2 resonance } & 17\end{array}$

$\begin{array}{lll}6 & \text { Conclusions } & 17\end{array}$

$\begin{array}{lr}\mathrm{A} \text { SO(6)/SO(5) model } & 19\end{array}$

$\begin{array}{lll}\text { A.1 The gauge sector } & 19\end{array}$

A.2 The fermion sector 20

\section{Motivation}

With the start of the second run of the Large Hadron Collider (LHC), we are seeing the first glimpses into physics at collision energies of $13 \mathrm{TeV}$. So far ATLAS and CMS have only collected a small amount of data $\left(3.2 \mathrm{fb}^{-1}\right.$ and $2.6 \mathrm{fb}^{-1}$ respectively), but that is already enough to set competitive limits on certain classes of new particles. For instance, jets and missing energy searches are already setting stronger limits on gluinos than at $8 \mathrm{TeV}$, due to the quickly growing parton luminosities at high masses. For new particles at lower masses, however, the parton luminosity increase is much milder and in most cases the $13 \mathrm{TeV}$ searches have not yet surpassed the $8 \mathrm{TeV}$ searches in sensitivity.

One $13 \mathrm{TeV}$ search that has received significant attention recently is the diphoton resonance search. Both ATLAS [1] and CMS [2] observe an excess at $750 \mathrm{GeV}$. It appears that the excess is compatible both with Run 1 data and between ATLAS and CMS which makes this a compelling case of potential new physics. In this paper we explore the model building possibilities to describe this excess from two complementary perspectives. The first perspective we take is to quantitatively analyze the low energy interactions needed to produce the observed diphoton rate. For this we identify the diphoton resonance as a new pseudoscalar particle that couples to the standard model (SM) through dimension 5 operators. The generic picture is that the pseudoscalar is produced in gluon fusion and then decays to a pair of photons. We show that this parametrization can account for the excess 
either with a large enhancement in the coupling to photons or with a moderate enhancement in the coupling to photons and gluons and moderate suppression in the couplings to fermions. Either of these cases implies that more particles in addition to the $750 \mathrm{GeV}$ resonance are needed to fit the data.

The second aspect of the diphoton excess that we address is naturally finding a scalar (or pseudoscalar) with a mass of $\sim 750 \mathrm{GeV}$ in a complete model. We know from the familiar example of the Higgs that theories with fundamental scalars appearing much below the cutoff are finetuned. One way this finetuning problem has been addressed is to posit that the Higgs is actually a composite particle of some new strong dynamics. While this idea solves the hierarchy problem in principle, in practice there is still residual tuning associated with a light Higgs meaning that we are forced to live with some level of tuning. It could be case, however, that there are other scalars coming from the strong dynamics that are not tuned. In other words, it could be that the Higgs as a pseudo Nambu Goldstone Boson (pNGB) is slightly tuned, but that the other pNGBs are at their naturalness limit. We will argue that in the composite Higgs framework one can have additional light scalars at the $750 \mathrm{GeV}$ mass scale and that such (pseudo)scalars are compatible with the excess.

Given the minimal information about the diphoton resonance, one cannot conclusively associate the resonance to the pseudoscalar parametrization that we present. We therefore survey a few other model building possibilities along with a few simple estimates to assess how easily these alternative models can fit the excess in comparison to the pseudoscalar case. In particular we look at a scalar resonance and a spin-2 resonance.

The outline is as follows. In section 2 we review the current experiment status of the diphoton resonance and collect limits from other potentially relevant channels. The interactions of a pseudoscalar are described in section 3 along with computations of widths, branching ratios, and rates. In section 4 we address the issue of getting the $\sim 750 \mathrm{GeV}$ mass scale in composite Higgs models. To conclude, in section 5 we point out other possibilities and summarize in section 6. Appendix A provides details of the $\mathrm{SO}(6) / \mathrm{SO}(5)$ composite Higgs model that contains a pseudoscalar pNGB.

A number of other works have studied the new physics implications of the $750 \mathrm{GeV}$ excess. These studies have looked at the effective theory for a spin-0 resonance [3-5], the effective theory including an additional field for dark matter $[6,7]$, adding vector-like quarks to enhance the interactions of the resonance with photons and gluons [8,9], adding a confining sector that is vector-like relative to the standard model $[10,11]$, and considering the resonance as an axion [12]. In terms of concrete models, refs. [3, 4] have pointed out that a scalar resonance from the composite sector of composite Higgs models could be the $750 \mathrm{GeV}$ resonance. These works are complementary to this paper in which, together with a model independent parametrization for the pseudoscalar, scalar, and spin-2 scenarios, we consider the Goldstone case in detail and explicitly identify regions of parameter space where composite models can account for the excess and the mass of the resonance can be naturally explained. They also agree with our conclusion that new particles in addition to the $750 \mathrm{GeV}$ resonance are required. 


\begin{tabular}{|c|ccc|}
\hline$\gamma \gamma$ & expected & observed & \\
\hline ATLAS (spin-2) & $1.9 \mathrm{fb}$ & $2.4 \mathrm{fb}$ & {$[13]$} \\
CMS (spin-2) & $1.5 \mathrm{fb}$ & $1.9 \mathrm{fb}$ & {$[14]$} \\
CMS (narrow) & $0.7 \mathrm{fb}$ & $1.3 \mathrm{fb}$ & {$[15]$} \\
CMS (wide) & $2.0 \mathrm{fb}$ & $2.3 \mathrm{fb}$ & {$[15]$} \\
\hline
\end{tabular}

Table 1. Upper limits (at $95 \% \mathrm{CL}$ ) on the $\sigma \times \mathcal{B R}$ of a $750 \mathrm{GeV}$ resonance decaying to a pair of photons from $8 \mathrm{TeV}$ LHC data.

\section{Signals and constraints from the LHC}

ATLAS and CMS have both reported excess in the diphoton channel at a mass very near to $750 \mathrm{GeV}$. For a narrow resonance, the local significance reported by ATLAS was $3.6 \sigma$ and $2.6 \sigma$ by CMS. When a wide resonance signal model is used, the significances shift to $3.9 \sigma$ for ATLAS and $2.0 \sigma$ for CMS. In ATLAS the global significance is $2.0 \sigma$ and in CMS it is $1.2 \sigma$.

To gain some idea of the expected sensitivity, we compile the expected and observed limits set by Run 1 diphoton searches in table 1 .

While a proper analysis should perform a combination of both the $8 \mathrm{TeV}$ and $13 \mathrm{TeV}$ results from both experiments to assess the compatibility of the signal and the correct cross section to fit, this is difficult to do reliably with such a small number of events. As such we will show the cross sections that can be obtained with a pseudoscalar resonance rather than fixing a signal strength value. As a guide, one can use the CMS combination of their $8 \mathrm{TeV}$ and $13 \mathrm{TeV}$ results which finds a cross section of $\sim 3-5 \mathrm{fb}$ [16].

In table 2 we list the observed limits from other channels that can be applicable to models that explain the diphoton excess. The limits shown are the observed limits and are set on $\sigma \times \mathcal{B R}$. For dijet limits we use the reported acceptance of $\mathcal{A}=0.6$ for spin- 0 signals to cast the limit from $\sigma \times \mathcal{B R} \times \mathcal{A}$ to $\sigma \times \mathcal{B R}[26,27]$. There are also searches for resonances in the $\tau^{+} \tau^{-}[32,33], Z h$ [34], and monojet [35, 36] channels which can be relevant for particular models.

In table 3 we rescale the strongest $8 \mathrm{TeV}$ limits by their $g g$ parton luminosity ratio [37] because in the models we consider the production is dominated by gluon fusion. A strict comparison of compatibility of a proposed model with $8 \mathrm{TeV}$ limits would involve simulating the signal model at $8 \mathrm{TeV}$ but the numbers in table 3 offer a quick comparison. Production from $q \bar{q}$ is suppressed by the fermion Yukawa couplings. For an example of a model in which the resonance is produced in $q \bar{q}$, see [38].

Finally, we note that the observed signal rate of $\sim 3-5 \mathrm{fb}$ is rather large. In the case of the SM Higgs, the decays to photons are mediated by loops of tops and $W$ 's and lead to a diphoton branching ratio of $\sim 10^{-3}$. If the decays of the $750 \mathrm{GeV}$ resonance to photons were likewise only mediated by tops and $W$ 's the diphoton ratio would be small, $\lesssim 10^{-5}$ (because $W W$ and $Z Z$ decays are now onshell), which would result in rates to $t \bar{t}$ and $W W$ of $\simeq 1000 \mathrm{pb}$ and $500 \mathrm{pb}$, respectively, at Run 1 . From table 2 this is clearly ruled out. Thus one can conclude that for a sufficiently large diphoton rate the $750 \mathrm{GeV}$ is not the only new particle, more are needed! 


\begin{tabular}{|cc|cc|}
\hline final state & & observed & \\
\hline$t \bar{t}$ & scalar & $700 \mathrm{fb}$ & ATLAS [17] \\
$t \bar{t}$ & spin-2 & $540 \mathrm{fb}$ & ATLAS [17] \\
$t \bar{t}$ & narrow & $450 \mathrm{fb}$ & CMS [18] \\
$t \bar{t}$ & wide & $510 \mathrm{fb}$ & CMS [18] \\
\hline$b \bar{b}$ & & $1.2 \mathrm{pb}$ & CMS [19] \\
\hline$Z \gamma$ & & $2.7 \mathrm{fb}$ & ATLAS [20] \\
\hline$Z Z$ & scalar & $12 \mathrm{fb}$ & ATLAS [21] \\
$Z Z$ & spin-2 & $38 \mathrm{fb}$ & ATLAS [22] \\
$Z Z$ & scalar & $23 \mathrm{fb}$ & CMS [23] \\
$Z Z$ & spin-2 & $53 \mathrm{fb}$ & CMS [24] \\
\hline$W W$ & spin-2 & $67 \mathrm{fb}$ & ATLAS [25] \\
$W W$ & scalar & $47 \mathrm{fb}$ & CMS [23] \\
\hline$j j$ & Gaussian & $2.0 \mathrm{pb}$ & ATLAS [26] \\
$j j$ & Breit Wigner & $20.0 \mathrm{pb}$ & ATLAS [26] \\
$j j$ & & $2.9 \mathrm{pb}$ & CMS [27] \\
\hline$\ell^{+} \ell^{-}$ & spin-2 & $1.1 \mathrm{fb}$ & ATLAS [28] \\
$\ell^{+} \ell^{-}$ & spin-2 & $3.5 \mathrm{fb}$ & CMS [29] \\
\hline$h h$ & & $32 \mathrm{fb}$ & ATLAS [30] \\
$h h$ & scalar & $51 \mathrm{fb}$ & CMS [31] \\
$h h$ & spin-2 & $39 \mathrm{fb}$ & CMS [31] \\
\hline
\end{tabular}

Table 2. Observed upper limits (at $95 \% \mathrm{CL}$ ) on $\sigma \times \mathcal{B R}$ of a $750 \mathrm{GeV}$ resonance decaying to various final states from $8 \mathrm{TeV}$ LHC data.

\begin{tabular}{|c|c|}
\hline final state & scaled \\
\hline$t \bar{t}$ & $2.1 \mathrm{pb}$ \\
$b \bar{b}$ & $5.6 \mathrm{pb}$ \\
$Z \gamma$ & $13 \mathrm{fb}$ \\
$Z Z$ & $56 \mathrm{fb}$ \\
$W W$ & $220 \mathrm{fb}$ \\
$j j$ & $9.4 \mathrm{pb}$ \\
$\ell^{+} \ell^{-}$ & $5.2 \mathrm{fb}$ \\
$h h$ & $150 \mathrm{fb}$ \\
\hline
\end{tabular}

Table 3. Observed LHC limits at $13 \mathrm{TeV}$ on $\sigma \times \mathcal{B R}$ rescaled from $8 \mathrm{TeV}$ using the $g g$ parton luminosity [37].

\section{The interactions of a pseudoscalar}

A spin-0 particle can either be a scalar or a pseudoscalar. The simplest possibility to start with is to consider an SM singlet. A scalar singlet can potentially mix with the Higgs which would introduce tree level decays to $t \bar{t}, W W, Z Z$ and even $h h$, which can place strong constraints on the mixing. It also suppresses the rate to photons compared to $V V$ 
similarly to the case of a heavy SM Higgs of mass $750 \mathrm{GeV}$. This very fact together with the relative importance of the diboson channels (see table 3) requires a huge contribution to the diphoton rate from new physics or a tuning of the mixing. Assuming CP conservation, a pseudoscalar will not mix with the Higgs which makes explaining the excess easier. ${ }^{1}$ We will therefore focus our discussion on a pseudoscalar resonance, and reserve comments on the scalar case until section 5 .

We consider the SM extended by the addition of an SM singlet pseudoscalar $\eta$ which transforms under $\mathrm{CP}$ as

$$
\eta \stackrel{\mathrm{CP}}{\longrightarrow}-\eta
$$

The scalar potential is given by

$$
V=V_{\mathrm{SM}}+\frac{m_{\eta}^{2}}{2} \eta^{2}+\frac{\lambda_{\eta}}{4 !} \eta^{4}+\frac{\lambda_{\eta h}}{2} \eta^{2}|H|^{2}
$$

We assume that $\mathrm{CP}$ is conserved, which at the level of the scalar potential simply acts as a $Z_{2}$ symmetry on $\eta$. This forbids mixing with the Higgs. The difference between $Z_{2}$ and CP becomes apparent when one considers non-renormalizable interactions. At dimension 5 the only interactions involving $\eta$ are

$\mathcal{L}_{\text {int }}=\frac{y_{f}}{\Lambda_{f}} \eta\left(i \overline{f_{L}} H f_{R}+\right.$ h.c. $)+\frac{c_{B}}{\Lambda_{g}} \frac{g^{\prime 2}}{16 \pi^{2}} \eta B_{\mu \nu} \tilde{B}^{\mu \nu}+\frac{c_{W}}{\Lambda_{g}} \frac{g^{2}}{16 \pi^{2}} \eta W_{\mu \nu}^{a} \tilde{W}^{a \mu \nu}+\frac{c_{g}}{\Lambda_{g}} \frac{\alpha_{s}}{8 \pi} \eta G_{\mu \nu}^{a} \tilde{G}^{a \mu \nu}$,

where $y_{f}$ is the Yukawa coupling of the fermion $f$ and $c_{B}, c_{W}$, and $c_{g}$ are parameters. For simplicity we supress all fermion operators by a common scale $\Lambda_{f}$ and all gauge field operators by a common scale $\Lambda_{g}$. These scales can of course be different and it is straightforward to generalize eq. (3.3). ${ }^{2}$ The normalization we use is $\tilde{B}^{\mu \nu}=\epsilon^{\mu \nu \alpha \beta} B_{\alpha \beta}$.

Notice that loops of SM fermions will already contribute to the interactions between the pseudoscalar and gauge boson pairs. The parameters $c_{B}, c_{W}$, and $c_{g}$ in eq. (3.3) denote contributions in addition to those from SM loops. As we are particularly interested in the diphoton rate, we define the parameter $c_{\gamma}=c_{B}+c_{W}$ which denotes the additional UV contribution to $\eta F_{\mu \nu} \tilde{F}^{\mu \nu}$. Notice that the relative sign between $c_{B}$ and $c_{W}$ depends on the details of the UV physics. Moreover, there is a physical sign between the SM top loop contribution and the $c_{i}$ coefficients.

One possibility for UV physics that could generate the dimension 5 operators above are heavy vector-like particles. In this case, one needs to be careful that the new particles do not lead to additional signals that would rule out the pseudoscalar explanation. For instance, requiring the new particles to be heavier than half the pseudoscalar mass protects against large branching ratios to these new particles. The limit where the new particles are just above threshold is interesting as the loop functions are maximal at threshold and could lead to sizable effects. In this work, these effects are parametrized with the aforementioned operator coefficients.

\footnotetext{
${ }^{1} \mathrm{~A}$ scalar as part of an additional doublet is another scenario that can be safe from mixing with the Higgs.

${ }^{2}$ Although one should note that in our parametrization the difference in gauge scales can be absorbed into $c_{B}, c_{W}$, and $c_{g}$.
} 

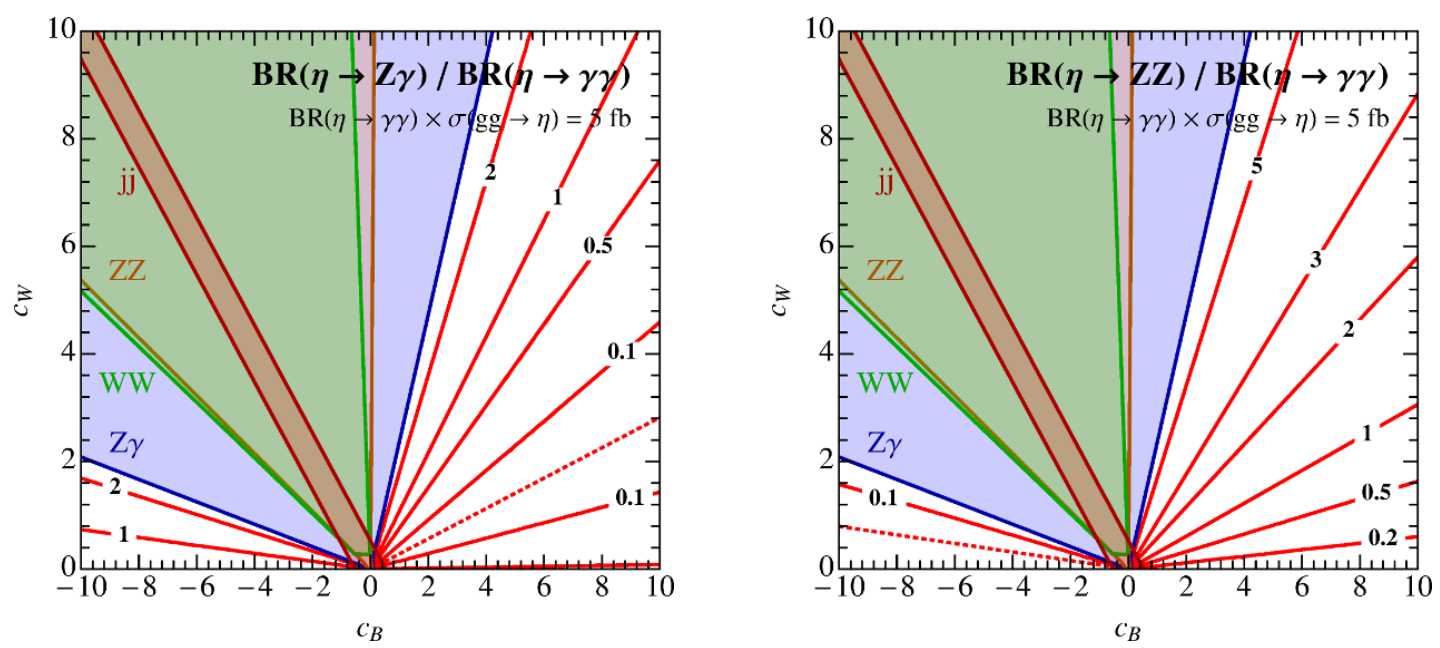

Figure 1. Branching ratio to $Z \gamma$ (left) and $Z Z$ (right) normalized to the diphoton branching ratio. The signal rate is fixed to $5 \mathrm{fb}$ and the blue, red, and green regions are excluded by $8 \mathrm{TeV}$ diboson searches while the brown region is excluded by dijet searches. The dotted red line shows where the branching ratio vanishes.

After fixing the pseudoscalar mass to $750 \mathrm{GeV}$, the parameter space consists of two dimensionful parameters $\Lambda_{f}$ and $\Lambda_{g}$ and three dimensionless parameters $c_{B}, c_{W}$, and $c_{g}$. One can see from the fact that $c_{\gamma}=c_{B}+c_{W}$ that the diphoton coupling can be increased by enhancing either the $\eta B_{\mu \nu} \tilde{B}^{\mu \nu}$ operators or the $\eta W_{\mu \nu}^{a} \tilde{W}^{a \mu \nu}$ operator. Increasing $c_{W}$ will increase the $W W$ coupling as well. In this work we set $c_{W}=0$ for simplicity such that branching ratio to $W W$ vanishes and $W W$ resonance searches are not constraining. The parameter space is $\left(\Lambda_{f}, \Lambda_{g}, c_{\gamma}, c_{g}\right)$.

It is also interesting to study the case where $c_{W} \neq 0$. When this is the case, $W W$ resonances searches become constraining in addition to constraints already from $Z \gamma$ and $Z Z$. While one can select combinations of $c_{B}$ and $c_{W}$ to set any of the branching ratios to $Z \gamma, Z Z$, or $W W$, to zero, the other two are necessarily non zero. In this sense, a generic prediction of the diphoton signal is a signal in two or more of the corresponding diboson channels. In figure 1 we show the branching ratio to $Z \gamma$ (left) and $Z Z$ (right) normalized to the diphoton branching ratio. In the plot $\Lambda_{f}$ is decoupled and consequently $t \bar{t}$ searches are not relevant.

Partial widths. Given the interactions in eq. (3.3) we can compute the partial decay widths. We only show the most relevant which are $t \bar{t}, g g, \gamma \gamma$, and to a lesser extent, $b \bar{b}$.

$$
\begin{aligned}
\Gamma_{t \bar{t}} & =\frac{N_{c}}{8 \pi} \frac{m_{t}^{2}}{\Lambda_{f}^{2}} m_{\eta} \sqrt{1-\frac{4 m_{t}^{2}}{m_{\eta}^{2}}}, \\
\Gamma_{b \bar{b}} & =\frac{N_{c}}{8 \pi} \frac{m_{b}^{2}}{\Lambda_{f}^{2}} m_{\eta} \sqrt{1-\frac{4 m_{b}^{2}}{m_{\eta}^{2}}},
\end{aligned}
$$



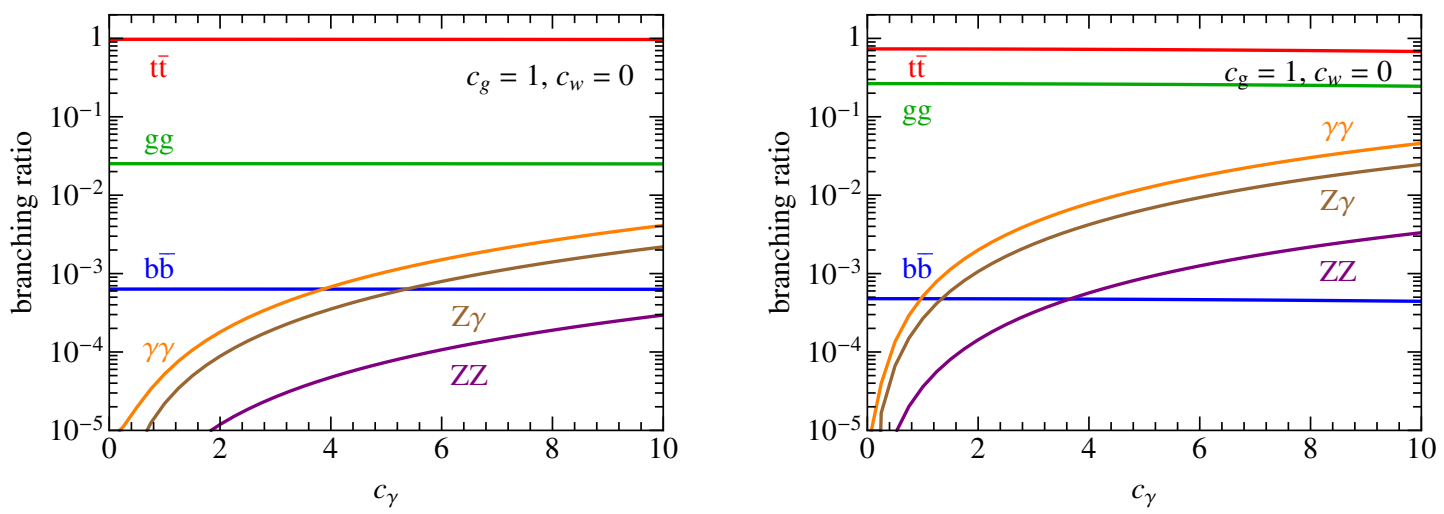

Figure 2. Branching ratios of the pseudoscalar as a function of $c_{\gamma}$ which parameterizes UV contributions to the pseudoscalar-photon-photon interactions. The parameters used are $\Lambda_{g}=500 \mathrm{GeV}$ and $\Lambda_{f}=750 \mathrm{GeV}$ (left) and $\Lambda_{f}=3 \mathrm{TeV}$ (right).

$$
\begin{aligned}
& \Gamma_{g g}=\frac{1}{2 \pi}\left(\frac{\alpha_{s}}{4 \pi}\right)^{2} \frac{m_{\eta}^{3}}{\Lambda_{f}^{2}}\left|A_{-}(\tau)+2 c_{g} \frac{\Lambda_{f}}{\Lambda_{g}}\right|^{2}, \\
& \Gamma_{\gamma \gamma}=\frac{1}{4 \pi}\left(\frac{\alpha}{4 \pi}\right)^{2} \frac{m_{\eta}^{3}}{\Lambda_{f}^{2}}\left|N_{c} Q_{t}^{2} A_{-}(\tau)+2 c_{\gamma} \frac{\Lambda_{f}}{\Lambda_{g}}\right|^{2},
\end{aligned}
$$

where $A_{-}(\tau)$ is the pseudoscalar loop function

$$
A_{-}(\tau)=\tau f(\tau), \quad \tau=\frac{4 m_{f}^{2}}{m_{\eta}^{2}},
$$

and the function $f(\tau)$ is given by

$$
f(\tau)=\theta(\tau-1) \arcsin ^{2}\left(\frac{1}{\sqrt{\tau}}\right)-\theta(1-\tau) \frac{1}{4}\left(\log \frac{1+\sqrt{1-\tau}}{1-\sqrt{1-\tau}}-i \pi\right)^{2} .
$$

The branching ratios to $Z \gamma, W W$, and $Z Z$ are correlated due to $\mathrm{SU}(2)$ gauge invariance. In the limit where we neglect the top loop (which is appropriate in the relevant parameter space) and $c_{W}=0$ the ratios $\operatorname{are}^{3}$

$$
\mathcal{B R}(\gamma \gamma): \mathcal{B R}(Z \gamma): \mathcal{B R}(Z Z): \mathcal{B R}(W W)=1: 2 t_{w}^{2}: t_{w}^{4}: 0
$$

From table 3 one can see that for the appropriate diphoton signal, none of the diboson channels are constraining.

In figure 2 we show the branching ratios as a function of $c_{\gamma}$ for two values of $\Lambda_{f}=$ $750 \mathrm{GeV}$ and $\Lambda_{f}=3 \mathrm{TeV}$ while $\Lambda_{g}=500 \mathrm{GeV}$ and $c_{g}=1$. We see that $t \bar{t}$ dominates the branching ratio unless it is supressed by a very large $\Lambda_{f}$ value. The branching ratios to $Z \gamma$ and $Z Z$ are estimated by only including their contribution from $c_{\gamma}$ and neglecting the top loop contribution to their partial widths.

From eq. (3.4) one can quickly estimate the width to be

$$
\frac{\Gamma}{m_{\eta}} \simeq \frac{N_{c}}{8 \pi} \frac{m_{t}^{2}}{\Lambda_{f}^{2}} \simeq 10^{-2}\left(\frac{600 \mathrm{GeV}}{\Lambda_{f}}\right)^{2} .
$$

The pseudoscalar tends to be narrow especially when $\Lambda_{f}$ becomes very large.

\footnotetext{
${ }^{3}$ Recall that this only holds when $c_{W}=0$.
} 

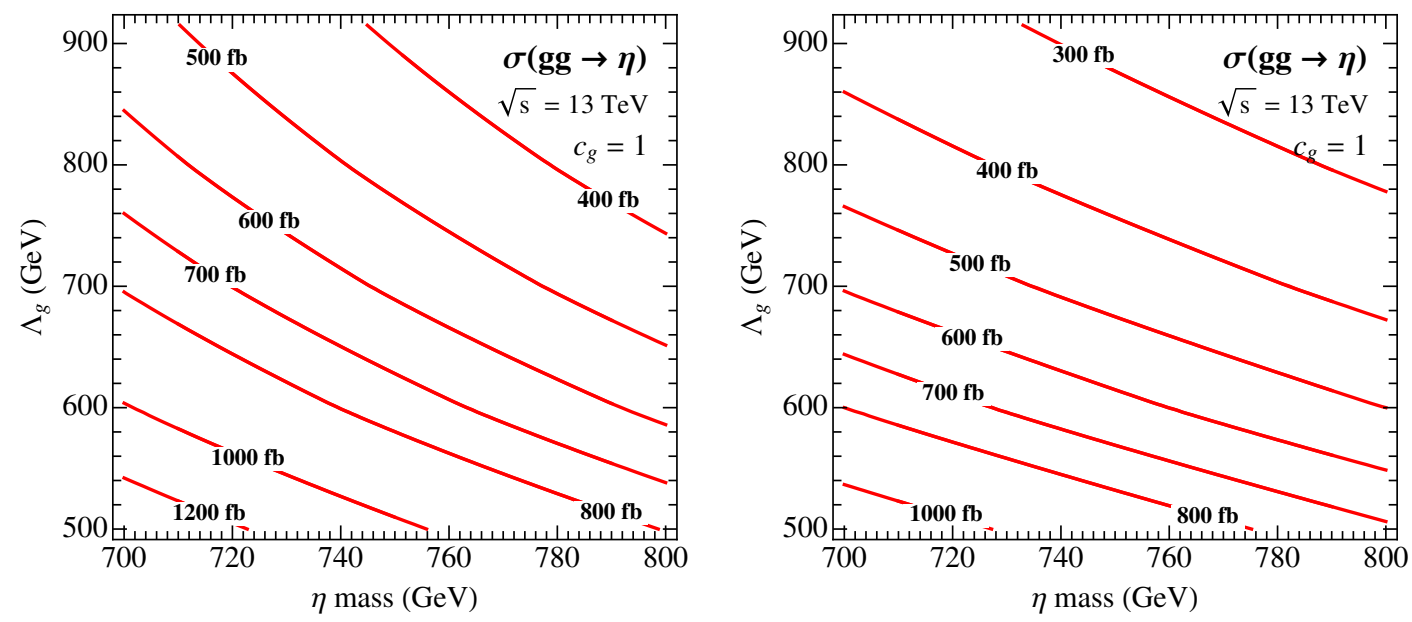

Figure 3. The production cross section of the pseudoscalar as a function of the mass and $\Lambda_{g}$ using $\Lambda_{f}=750 \mathrm{GeV}$ (left) and $\Lambda_{f}=3 \mathrm{TeV}$ (right).

Production rate. From the branching ratios, one can see that the $\eta$ is produced in gluon fusion. We show the total production cross section as a function of mass and the gauge field scale $\Lambda_{g}$ in figure 3 for $\Lambda_{f}=750 \mathrm{GeV}$ (left) and $\Lambda_{f}=3 \mathrm{TeV}$ (right). The only SM fermion we include in the loop is the top quark. We compute the pseudoscalar cross section at NNLO using HIGLU [39] and rescale the cross section to account for an additional gluon fusion contribution via $c_{g}=1$. The value of $\Lambda_{g}$ controls the relative rate due to the additional dimension 5 contribution.

Simple estimates of the production rate are useful and straightforward to obtain using information provided by the Higgs working group [40] which provides the production rates for heavy Higgses produced in gluon fusion as a function of mass at $8 \mathrm{TeV}$. First, one needs to account for the difference between scalar and pseudoscalar production. At leading order difference can be obtained by the ratio of loop functions

$$
\sigma_{\eta}=\left|\frac{3}{2} \frac{A_{-}\left(\frac{4 m_{t}^{2}}{m_{\eta}^{2}}\right)}{A_{+}\left(\frac{4 m_{t}^{2}}{m_{\eta}^{2}}\right)}\right|^{2} \sigma_{H}
$$

where $A_{+}(\tau)$ is the scalar loop function

$$
A_{+}(\tau)=\frac{3}{2} \tau(1+(1-\tau) f(\tau))
$$

At $750 \mathrm{GeV}$ this ratio works out to be $\simeq 1.45$. Next, one can rescale the $8 \mathrm{TeV}$ rates to $13 \mathrm{TeV}$ by the parton luminosities which is 4.7 for a $g g$ initial state [37]. Finally one needs to account for the prefactor of the pseudoscalar-top coupling in eq. (3.3) relative to the Higgs-top coupling in the standard model. Compiling these numbers together and rescaling from the NNLL QCD + NLO electroweak $8 \mathrm{TeV}$ rate, one finds the rate at $13 \mathrm{TeV}$ to be $\left(\right.$ for $c_{g}=0$ )

$$
\left.\sigma_{\eta}(750 \mathrm{GeV})\right|_{c_{g}=0}=\left(\frac{v}{\Lambda_{f}}\right)^{2} \times 1.0 \mathrm{pb} .
$$




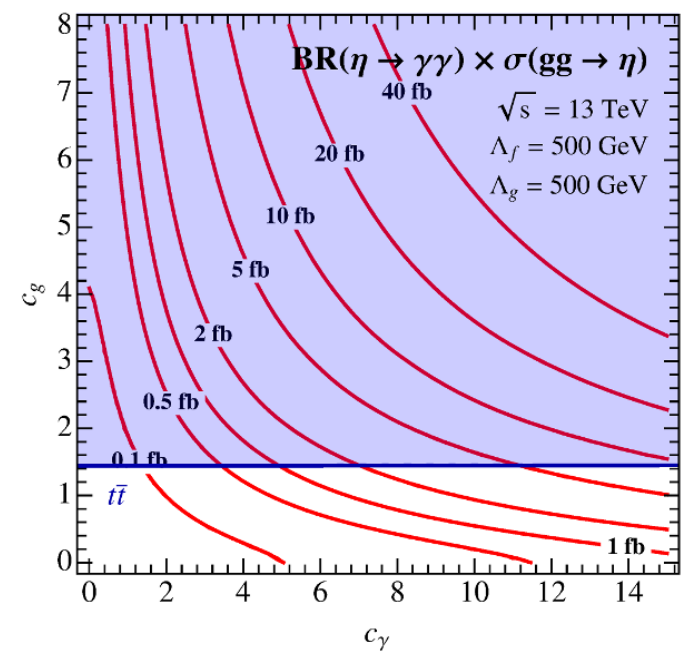

Figure 4. The diphoton rate at $13 \mathrm{TeV}$ using $\Lambda_{f}=500 \mathrm{GeV}$ and $\Lambda_{g}=500 \mathrm{GeV}$. The blue region is excluded by $t \bar{t}$ searches.

Therefore given a mild suppression from $\left(v / \Lambda_{f}\right)^{2}$ and a diphoton branching ratio of $\sim$ $10^{-3}-10^{-2}$ one can see that the diphoton rate will be $\mathcal{O}(5 \mathrm{fb})$ as is needed to explain the excess. Allowing for a non vanishing $c_{g}$ the above result is then rescaled (and typically enhanced) by the ratio of the partial width to gluons in the two cases, $\Gamma_{g g}\left(c_{g}\right) / \Gamma_{g g}\left(c_{g}=0\right)$.

The diphoton rate is computed in figure 4 as a function of $c_{\gamma}$ and $c_{g}$. Clearly $c_{\gamma}$ only affects the branching ratio, while $c_{g}$ both the total rate and the branching ratio since it modifies $\Gamma_{g g}$. The blue shaded region indicates where the model is ruled out by $8 \mathrm{TeV}$ searches for $t \bar{t}$ resonances (rescaled to $13 \mathrm{TeV}$ ). One can see that a sufficient diphoton rate can be achieved by having either one of $c_{\gamma}$ or $c_{g}$ to be sizable, but because $c_{g}$ increases the total rate, the $t \bar{t}$ rate also increases. Dijet searches also constrain $c_{g}<6$ and $Z \gamma$ searches constrain the diphoton rate to be less than $20 \mathrm{fb}$. These are not shown in figure 4 since $t \bar{t}$ is stronger than both.

In figure 5 we slice the parameter space differently and fix a small contribution to gluon fusion via $c_{g}=2$ and look at the dependence on $\Lambda_{f}$. We see that as $\Lambda_{f}$ is increased, the top loop contribution to the production shrinks as does the $t \bar{t}$ rate itself. For a negative value of $c_{g}$ we can have destructive interference with the top loop contribution. In this paper, we do not consider this possibility. The appropriate rate is still attainable from the $c_{g}$ and $c_{\gamma}$ contributions. Dijet searches are not constraining here because the overall rate is smaller and $Z \gamma$ searches still bound the overall diphoton rate (but is not shown in figure 5).

Results. From figures 4 and 5 one can see that it is possible to achieve the observed signal rate of $\sim 3-5 \mathrm{fb}$. In both cases the strongest constraints come from $t \bar{t}$. Dijet searches are not as sensitive nor are diboson searches as we have used safe value of $c_{W}=0$. From the interplay of the effective operators of eq. (3.3) two parameter regions that can explain the excess can be identified:

- A single scale where $\Lambda_{f}=\Lambda_{g}=f$ as in figure 4. Given that the scales are not too large, the pseudoscalar to gluon coupling must come mainly from the top loop and one requires a large $c_{\gamma}$ value to get the diphoton rate. 


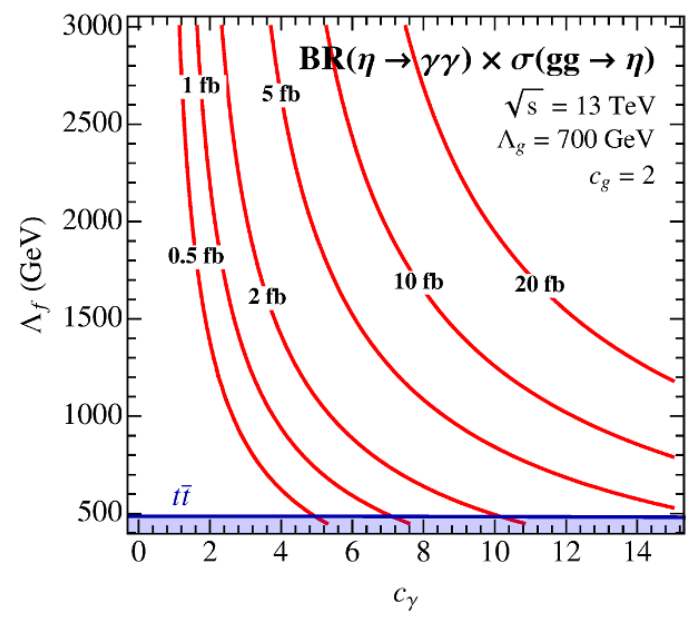

Figure 5. The diphoton rate at $13 \mathrm{TeV}$ using $\Lambda_{g}=700 \mathrm{GeV}$ and $c_{g}=2$. The blue region is excluded by $t \bar{t}$ searches.

- Suppressed fermions where $\Lambda_{f} \gg \Lambda_{g}=f$ as in figure 5. Here the pseudoscalar to top coupling is small enough that $t \bar{t}$ searches are not too constraining. Then gluon fusion can receive a moderate enhancement and the pseudoscalar to photon coupling also only needs a moderate enhancement.

We use the scale $f$ to indicate the scale at which the dimension 5 operators are generated. In the case of a suppressed fermion contribution one can achieve $\Lambda_{f} \gg \Lambda_{g}$ either by the fermion contribution being generated at a much higher scale or by a small prefactor such that $\Lambda_{f} \gg f$. The latter case will be relevant for the composite Higgs case. Due to the interplay between the top loop and the contribution from the effective operator, we focus on $c_{g}=1-2$. This value can be achieved with a colored fermion at a mass scale of $\approx 400 \mathrm{GeV}$, or several colored fermions at higher masses. Another possibility is the presence of anomaly induced contributions to the $\eta$ effective couplings to gauge bosons (for a discussion see [4]).

Another possibility for a large enough diphoton rate, not mentioned above, is to invoke a large contribution from $c_{g}$. To avoid $t \bar{t}$ bounds, it is needed that $\Lambda_{f} \gg \Lambda_{g}$, making the coupling of $\eta$ to tops negligible for all practical purposes. In this limit the rate no longer depends on $\Lambda_{f}$, but only on $\left(\Lambda_{g}, c_{\gamma}, c_{g}\right)$. If, for illustration, one fixes $\Lambda_{g}=700 \mathrm{GeV}$ and the diphoton rate to $2 \mathrm{fb}$, then $c_{g}$ is the only free parameter since $c_{\gamma}$ is determined by the diphoton rate. Then the dijet rate is $\approx c_{g}^{2}(400 \mathrm{fb})$ and the total width is $\approx c_{g}^{2}(0.04 \mathrm{GeV})$. We see that in this case, dijet searches bound $c_{g} \lesssim 4$ and produce a narrow resonance.

Now that we have identified viable regions of parameter space we comment on the width in more detail than eq. (3.8). Figure 6 shows the width as a function of $\Lambda_{f}$ and the invisible branching ratio. With only the SM states we have discussed, there is no invisible width and the $\eta$ tends to be narrow. A wider resonance can be obtained by adding an invisible width. In figure 6 the total rate is small enough that monojet searches do not yet constrain this parameter space. 

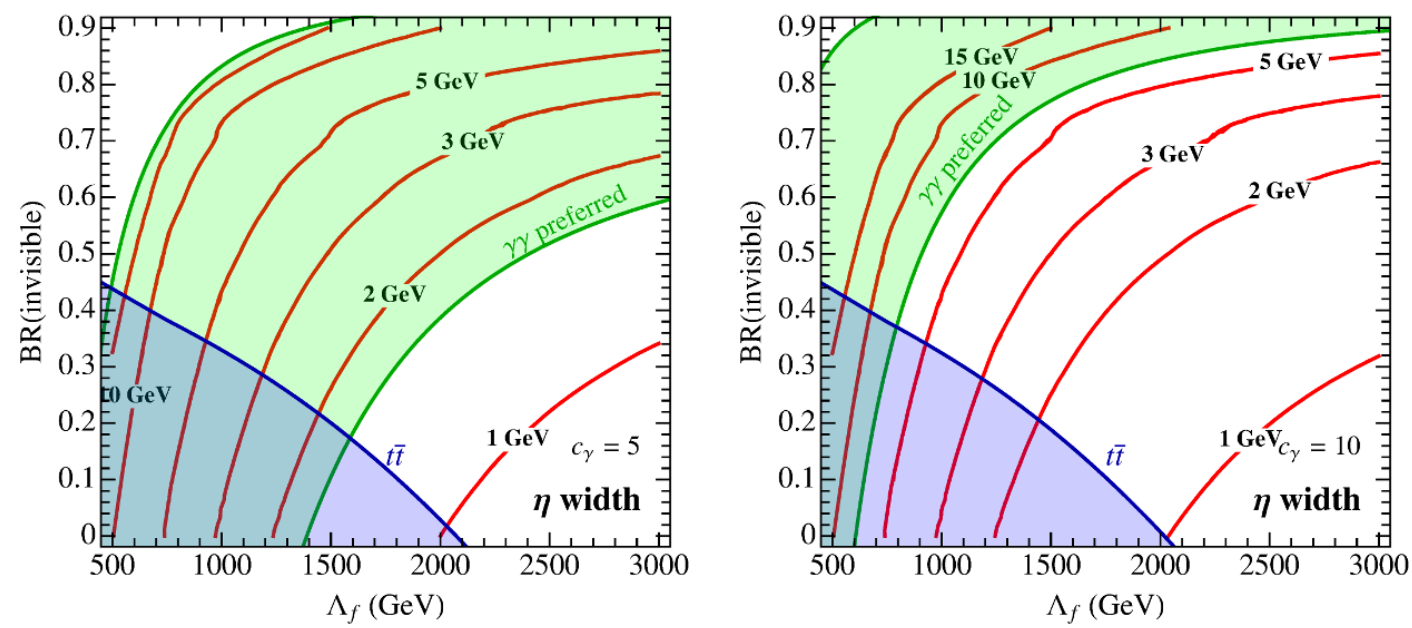

Figure 6. The width of the pseudoscalar as a function of the fermion suppresion scale $\Lambda_{f}$ and the invisible branching ratio for $c_{\gamma}=5$ (left) and $c_{\gamma}=10$ (right). The blue region is excluded by $t \bar{t}$ searches and the green region has a diphoton rate between 1 and $10 \mathrm{fb}$. The parameters used are $\Lambda_{g}=500 \mathrm{GeV}$ and $c_{g}=2$.

\section{The mass scale of a pseudoscalar}

In this section we describe a model in which one can expect to find a pseudoscalar of mass $\sim 750 \mathrm{GeV}$. In this model, both the Higgs and the $\eta$ are pNGBs of a global symmetry. The argument is based on the composite Higgs scenario (for a nice review, see [41]) where the lightest particles of the composite sector are pNGBs. The minimal case identifies the pNGB multiplet with the Higgs multiplet which crucially depends on the global symmetries [42]. One can consider non-minimal scenarios, however, where there are additional light pNGBs which can have various quantum numbers and could even be SM singlets. See [43-47] for previously studied examples.

The general framework. In adding another light scalar, where light is relative to the cutoff, one is once again faced with a hierarchy problem. Just as identifying the Higgs as a pNGB can explain its small mass, the presence of an additional $\sim 750 \mathrm{GeV}$ pseudoscalar can be naturally justified if it is also a pNGB of a global symmetry.

In order to accommodate an extra singlet (or extra singlets) we need to go beyond the minimal composite Higgs model [42] and consider a larger global group $\mathcal{G}$. The coset $\mathcal{G} / \mathcal{H}$ then contains the SM Higgs doublet and extra scalars. ${ }^{4}$ To control custodial breaking effects that may be induced by the additional scalars it is phenomenologically important to add extra discrete symmetries [47].

As we avoid discussion of a particular model, for our purposes it is sufficient to highlight a few generic facts for models with a pseudoscalar singlet pNGB in addition to the Higgs pNGB multiplet. The full set of pNGBs can be parametrized as

$$
U(\Pi)=\exp \left(\frac{i}{f}\left(\widehat{H}+\eta T_{\eta}+\ldots\right)\right),
$$

\footnotetext{
${ }^{4} \mathrm{~A}$ notable case is $\mathrm{SO}(6) / \mathrm{SO}(5)$ with a Higgs and a pseudoscalar singlet [43], see appendix A.
} 
where $\widehat{H}$ is a compact notation for the matrix of pNGBs that will be identified with the SM Higgs and $\eta$ is the pseudoscalar associated with the broken generator $T_{\eta}$. The $\ldots$ indicate additional pNGBs that could be present.

The standard model $\mathrm{SU}(2)_{L} \times \mathrm{U}(1)_{Y}$ gauges a subgroup of the unbroken $\mathcal{H}$. In particular, for $\eta$ to be a singlet, we must have

$$
\left[T_{\eta}, T_{\mathrm{SM}}\right]=0,
$$

where $T_{\mathrm{SM}}$ are the generators corresponding to the SM gauge fields. This has relevance for phenomenology, since as it is a singlet the $\eta$ does not couple to SM gauge fields.

The general couplings of the pNGBs to SM vectors are given by

$$
\frac{g_{h V V}}{g_{h V V}^{\mathrm{SM}}}=1-\kappa_{V} \frac{v^{2}}{f^{2}}+\mathcal{O}\left(\frac{v^{4}}{f^{4}}\right), \quad \frac{g_{\eta V V}}{g_{h V V}^{\mathrm{SM}}}=0,
$$

where $g_{h V V}^{\mathrm{SM}}$ is the Higgs-vector-vector coupling in the standard model and $\kappa_{V}$ is an $\mathcal{O}(1)$ coefficient. The pseudoscalar does not couple to SM vectors at tree level. From eq. (4.3) one can derive a lower bound on the scale $f$ which is found to be $f \gtrsim 600 \mathrm{GeV}$, which comes from measurements of Higgs couplings [48, 49]. Another important implication of eq. (4.3) is that the gauge interactions do not contribute to the one loop generation of a bare mass of the pseudoscalar.

The fermion sector. At this point the couplings between the pNGBs and the SM fermions have not been specified. In this work we focus primarily on the coupling of the pseudoscalar to the top quark because it has the largest Yukawa coupling. The usual generation of masses for SM quarks in composite Higgs models proceeds via the partial compositeness mechanism [50] where the elementary fields couple to operators from the composite sector. Schematically the coupling is

$$
y_{L} \overline{q_{L}} \cdot U \cdot \Psi+y_{R} \overline{u_{R}} \cdot U \cdot \Psi+\text { h.c. }
$$

where $\Psi$ represent composite operators and $y_{L}$ and $y_{R}$ are related to the fermion Yukawas. While eq. (4.4) can be made formally non linearly invariant under $\mathcal{G}$, the SM fermions are embedded in incomplete multiplets of $\mathcal{G}$ which breaks the global symmetries. This breaking in turn generates Yukawa couplings and a potential for the pNGBs. Generically, the Higgs potential always receives a contribution from at least the left handed mixing.

The interactions of the singlet, on the other hand, are model dependent. In particular, if the embeddings of $q_{L}$ and/or $u_{R}$ are not eigenstates of the generator $T_{\eta}$, then in general the interactions of eq. (4.4) break the shift symmetry of $\eta$ and contribute to its potential. It is also important to ensure that the embeddings are consistent with our assumption of $\mathrm{CP}$ conservation. It has been shown that this can be done in concrete examples [43].

By the appropriate insertions of spurions, $y_{L}$ and $y_{R}$, we can construct the would-be Yukawa term

$$
y_{t} \overline{t_{L}} h t_{R}\left(1+i \kappa_{\eta} \frac{\eta}{f}+\mathcal{O}\left(\frac{1}{f^{2}}\right)\right)+\text { h.c. }
$$

where $\kappa_{\eta}$ is an $\mathcal{O}(1)$ coefficient. 
The couplings of the $h$ and $\eta$ to top quarks is found to be

$$
\frac{g_{h t t}}{g_{h t t}^{\mathrm{SM}}}=1-\kappa_{F} \frac{v^{2}}{f^{2}}, \quad \frac{g_{\eta t t}}{g_{h t t}^{\mathrm{SM}}}=i \frac{v}{f} \kappa_{\eta} .
$$

where $g_{h t t}^{\mathrm{SM}}$ is the top coupling to the Higgs in the standard model and $\kappa_{F}$ is an $\mathcal{O}(1)$ coefficient that depends on the embedding of the fermions. Notice that derivation has been completely general, and the only assumptions have been related to the $\mathrm{CP}$ nature of the singlet. It is also manifest that, from the SM perspective, the coupling of the $\eta$ arises at dimension 5 in complete analogy with the simplified discussion of section 3.

Mass of the pseudoscalar. The mass of the $\eta$ is determined by the parameter that breaks its shift symmetry. Even though the $\eta$ is an SM singlet, if the embeddings of $q_{L}$ or $u_{R}$ break $T_{\eta}$, then the $\eta$ 's shift symmetry will be broken. Then eq. (4.5) will contribute to the $\eta$ 's mass via a contribution to $\lambda_{\eta h}$. This contribution is chirality breaking and involves a Higgs field. There is a chirality preserving contribution that we expect to directly contribute to $m_{\eta}^{2}$ and arises in the following way.

After having integrated out the composite sector at low energies for $u_{R}$ we have

$$
\overline{u_{R}} \not p u_{R}+y_{R}^{2} F_{u_{R}}\left(p^{2}, m_{*}\right) \overline{u_{R}} \not p u_{R}\left(c_{\eta} \frac{\eta^{2}}{f^{2}}+\ldots\right)
$$

where $F_{u_{R}}$ is a form factor that encodes the contribution of the resonances of the strong sector. The poles of $F_{u_{R}}$ correspond to the masses of the resonances of the strong sector. Here we use $m_{*}$ to denote the various mass scales of the resonances that we expect below $4 \pi f$, but above $f$.

Note that eq. (4.7) is generic for pNGBs that couple to $u_{R}$. It is possible that in specific models $c_{\eta}$ can vanish due to accidental symmetries $[43,47]$. In other models $c_{\eta}$ can be proportional to $\kappa_{\eta}$. Here we simply consider it to be an $\mathcal{O}(1)$ coefficient. We find a term in the effective potential of the form

$$
c_{\eta} \frac{N_{c} y_{R}^{2}}{4 \pi^{2}} m_{*}^{2} \eta^{2} .
$$

Fixing the top Yukawa, we find

$$
y_{t} \simeq \frac{f}{m_{*}} y_{L} y_{R}
$$

and taking $y_{L} \sim y_{R}$ we arrive at the estimate,

$$
m_{\eta}^{2} \simeq \frac{N_{c} y_{t}}{2 \pi^{2}} \frac{m_{*}^{3}}{f} .
$$

For reasonable values of the parameters we get the estimate,

$$
m_{\eta} \simeq 750 \mathrm{GeV}\left(\frac{m_{*}}{1.3 \mathrm{TeV}}\right)^{3 / 2}\left(\frac{600 \mathrm{GeV}}{f}\right)^{1 / 2} .
$$

Interestingly, this is of the right size. It is worth further emphasizing that this mass is at the naturalness limit for $\eta$ since no tuning is required. This result can benefit from further 
explanation. The estimate in eq. (4.11) can be obtained without tuning the mass of the singlet. However, there is a preliminary tuning, which is unavoidable in this context, and it is related to the tuning of the Higgs vacuum expectation value that needs to be $v \ll f$ to comply with the potentially dangerous precision constraints. There is also a tuning of the Higgs quartic, which is more model dependent and has a different scaling with the parameters of the model (see [41]). Once the tuning of $\mathcal{O}\left(v^{2} / f^{2}\right)$ has been achieved, then the other pNGB is at its natural limit. In conclusion, it is possible to argue that in the composite Higgs scenario there is no need to tune the mass of the singlet, and it is expected to be parametrically lighter than the cutoff due to its pNGB nature.

In this respect, we usually expect a ratio given by

$$
\frac{m_{\eta}}{m_{h}} \sim \sqrt{\frac{g_{*}}{y_{t}}} \frac{f}{v}
$$

Notice that the usual tuning in composite Higgs models requires $g_{*} \simeq m_{*} / f \simeq \mathcal{O}(1)$, i.e. top partners within reach of the LHC. The same prediction derived from the Higgs mass is true in this model from the $\eta$ mass. Models of this type, where the mass of the new resonance is technically natural and linked to the explanation of size of the Higgs mass, seem to deserve further attention even if one has to introduce new ingredients on top of the minimal models.

Interactions of the singlet. In order to connect the composite $\eta$ with the results of section 3 we comment on the size of $c_{\gamma} / \Lambda_{g}$. We start with the top coupling, which from eq. (4.6), tells us that tops will couple to the pseudoscalar with a $v / f$ suppression according to

$$
\frac{1}{\Lambda_{f}} \simeq \frac{\kappa_{\eta}}{f}
$$

In the limit where the $\eta$ is the lightest new state, the loop induced couplings to gluons and photons are dominated by top contributions.

In the composite sector there are particles (the top partners) charged under both SU(3) and electromagnetism that can also run in the loop. From the view of the composite sector, $\eta$ is a NGB which means that any shift breaking interaction with top partners must go through an elementary composite mixing. For an estimate, we note that each power of $\eta$ comes with at least one power of $y \sim y_{R} \sim y_{L}$. Given the symmetries of the strong sector, some of these corrections can have further suppressions. For estimates see appendix A.

The challenge of finding large enough $c_{\gamma}$ presents itself from the fact that top partner searches have been performed and it seems difficult to evade a bound of $\sim 700 \mathrm{GeV}$ (see for example [51,52]) and go into a region where the loop functions are enhanced. A similar scaling is expected for the top partner contribution to $c_{g}$ (without the color and electric charge factors).

It is possible that the global (non-linearly realized) symmetry of the strong group is anomalous. In the case where the generator associated to $\eta$ has non-vanishing anomaly coefficients with two SM gauge bosons, one can have dimension 5 operators in complete analogy with eq. (3.3). The simplest scenario with a light singlet, $\mathrm{SO}(6) \simeq \mathrm{SU}(4)$, can have global anomalies $\left(\mathrm{SU}(4)^{3}\right)$ although with $c_{\gamma}=0$ (see appendix A). 
Other coset spaces can have additional singlets, an example (that suffers custodial breaking and hence is tuned) is $\mathrm{SU}(3) \times \mathrm{U}(1)_{X} / \mathrm{SU}(2)_{L} \times \mathrm{U}(1)_{Y}[53,54]$. The NGBs are in the $\mathbf{2}_{ \pm 1 / 2}+\mathbf{1}_{0}$ of $\mathrm{SU}(2)_{L} \times \mathrm{U}(1)_{Y}$. In this case $T_{\eta} \sim \operatorname{diag}(0,0,1)$. The presence of the $\mathrm{U}(1)_{X}$ allows for a correct hypercharge assignment and the NGBs have charge $X=-1 / 3$. Hypercharge is defined as $Y=(1 / 2 \sqrt{3}) \lambda_{8}+X$, where $\lambda_{a}$ are the Gell-Mann matrices. In this case $T_{\mathrm{em}}^{2}$ has no particular structure and $\operatorname{tr}\left[T_{\eta} T_{\mathrm{em}}^{2}\right] \neq 0$ in general. At low energies this can manifest itself into an anomalous contribution in the form

$$
n_{\gamma} \frac{\alpha}{4 \pi} \frac{\eta}{f} F_{\mu \nu} \tilde{F}^{\mu \nu}
$$

which can help numerically to get a sizable decay to photon pairs. Other choices of global groups could give the same contribution (finding these groups could be a direction of further study), and more exotic groups can also contain color anomalies, hence a contribution to $c_{g}$. Finally, notice that in this case the anomaly coefficient is not suppressed by SM couplings.

Results. As a brief summary of the possibilities discussed, we comment on two specific cases, both using a moderate scale $f \sim 600 \mathrm{GeV}$, as is suggested by Higgs coupling measurements and naturalness considerations. In particular, given the notation of eq. (3.3) we consider $\Lambda_{g}=f$ and $\Lambda_{f}=f / \kappa_{\eta}$ where $\kappa_{\eta}$ is defined in eq. (4.6). We leave $c_{\gamma}$ and $c_{g}$ as free parameters, having in mind the possible size as suggested by the previous estimates.

- $\kappa_{\eta}$ is $\mathcal{O}(1)$. In this case where $\Lambda_{f}=\Lambda_{g}=f$ in order to sufficiently enhance the diphoton rate a large anomalous contribution to the diphoton coupling seems necessary.

- $\kappa_{\eta}$ is reduced. Then $\Lambda_{f}>\Lambda_{g}=f$ and we can be in the case where $\Lambda_{f} \simeq 3 \mathrm{TeV}$ where only moderate values of $c_{\gamma}$ and $c_{g}$ are required (see figure 5). This is probably still difficult to achieve in the minimal realizations of composite Higgs which only include tops and top partners.

Both of these cases can be visualized in figure 7, where we have fixed $c_{\gamma}=2$ and $c_{g}=2$. Near the top at $\kappa_{\eta} \simeq 1$ the rate is too low with $c_{\gamma}$ and $t \bar{t}$ forces $f$ to start to become large. For small $\kappa_{\eta}$ both the diphoton rate is sufficient and $f$ can be near the preferred value.

\section{Alternative explanations}

While we have focused on the case of a pseudoscalar resonance, there are obviously a number of possible explanations. The general obstacles that models face were also shared in the pseudoscalar case which are boosting the $\gamma \gamma$ branching ratio to $\sim 10^{-2}$ and avoiding constraints from $W W, Z Z, Z \gamma$, and $h h$ searches. In fact, a nice feature of the pseudoscalar explanation is that symmetries enforce a loop level coupling to both photons and transverse vector bosons, easily evading diboson constraints.

In this section, we briefly outline two other scenarios that could be plausible, namely a spin-0 scalar resonance and a spin-2 resonance. There are other scenarios one can envisage which we do not comment on at all. One example of this would be a $750 \mathrm{GeV}$ particle decaying to two $\mathcal{O}(100 \mathrm{MeV})$ particles that each decay to photon pairs. The large boost of the light particles then cause the pair of photons to be detected as a single photon. 


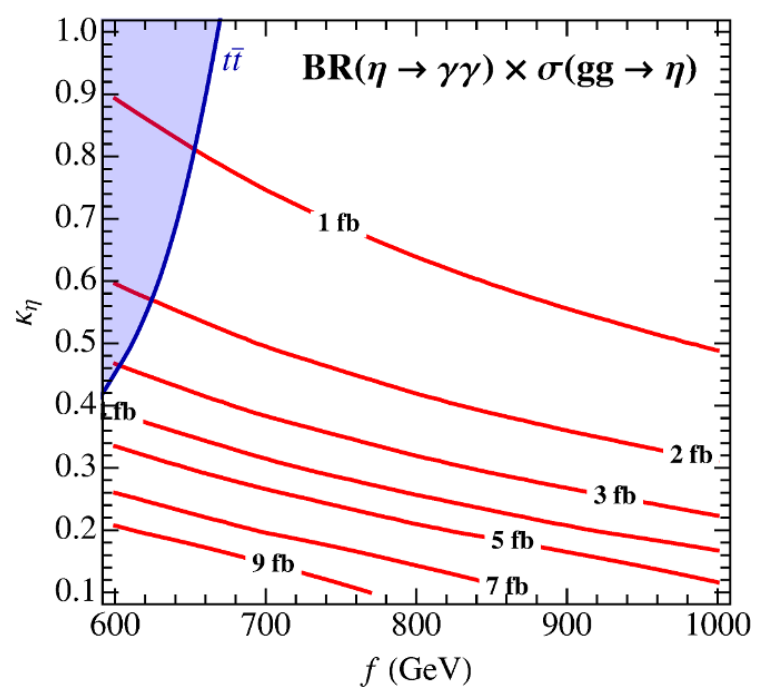

Figure 7. The diphoton rate at $13 \mathrm{TeV}$ for the composite Higgs scenario. The parameters $c_{g}=2$ and $c_{\gamma}=2$ are used.

\subsection{Scalar resonance}

A model very similar to the pseudoscalar is a scalar singlet $s$ added to the SM. While assuming CP allowed us to restrict the pseudoscalar from mixing with the SM, we do not have such a symmetry for the scalar (since $Z_{2}$ is not useful in this context). In any case, it is possible to assume that the only couplings of the scalar $s$ to the SM are through $F_{\mu \nu} F^{\mu \nu}$ and $G_{\mu \nu}^{a} G^{\mu \nu a}$, possibly induced by heavy vector-like fermions (see e.g. [52]). As discussed in section 3 we find it useful to work below the scale of the new fermions (in order to avoid decays to them). ${ }^{5}$ This model is parametrized by only two interactions (for simplicity, we neglect here constraints from $Z \gamma$, see section 2)

$$
\mathcal{L}=\frac{\alpha}{4 \pi} \frac{s}{\Lambda_{F}} F_{\mu \nu} F^{\mu \nu}+\frac{\alpha_{s}}{4 \pi} \frac{s}{\Lambda_{G}} G_{\mu \nu}^{a} G^{\mu \nu a} .
$$

The overall rate is a function of $\Lambda_{G}$ and can be estimated by rescaling the SM rate (similar to section 3 )

$$
\sigma_{s}(750 \mathrm{GeV})=\left(\frac{v}{\Lambda_{G}}\right)^{2} \times 1.25 \mathrm{pb} .
$$

The branching ratios are functions of both scales (and gauge couplings) and are

$$
\begin{aligned}
\mathcal{B R}_{s \rightarrow g g} & =\frac{8 \alpha_{s}^{2}}{8 \alpha_{s}^{2}+\alpha^{2}\left(\frac{\Lambda_{G}}{\Lambda_{F}}\right)^{2}}, \\
\mathcal{B R}_{s \rightarrow \gamma \gamma} & =\frac{\alpha^{2}\left(\frac{\Lambda_{G}}{\Lambda_{F}}\right)^{2}}{8 \alpha_{s}^{2}+\alpha^{2}\left(\frac{\Lambda_{G}}{\Lambda_{F}}\right)^{2}},
\end{aligned}
$$

\footnotetext{
${ }^{5}$ As the heavy fermions must be colored to couple to gluons, they also must be unstable because they are colored. Additional model building is necessary to ensure these are phenomenologically viable and will likely lead to specific predictions of the mass or couplings.
} 
which can give $\mathcal{B R}_{s \rightarrow \gamma \gamma} \sim 10^{-2}$ when $\Lambda_{G}$ is sufficiently larger than $\Lambda_{F}$. Then one needs to adjust the cross section with $\Lambda_{G}$ where the right value is $\Lambda_{G}^{2} / v^{2} \simeq 5$. A full analysis would involve a study of the heavy fermions, including their impact on the running of gauge and Yukawa couplings (which is especially important if the two loop induced effects on the Higgs quartic are large). Such studies are model dependent but would allow one to make concrete predictions for accompanying signals.

\section{$5.2 \quad$ Spin-2 resonance}

There are no obstructions for spin-2 particles to decay to photon pairs. Here we consider a hypothetical massive spin-2 particle $\rho_{\mu \nu}$ with a mass $m_{\rho}$ that couples to the SM stress energy tensor. While it is debatable whether such a light spin-2 particle could be the first observed state (e.g. this is not the case in QCD), we explore this possibility with a very pragmatic approach.

Consider a spin-2 Lagrangian with a Fierz-Pauli mass term (as is automatically implied by the Kaluza Klein reduction of 5 dimensional models, see [55] for a review)

$$
\mathcal{L}=\mathcal{L}_{\text {spin }-2}^{\mathrm{FP}}+\frac{1}{\Lambda_{G}} \rho_{\mu \nu} T_{B}^{\mu \nu}+\frac{1}{\Lambda_{F}} \rho_{\mu \nu} T_{F}^{\mu \nu},
$$

where $\rho_{\mu \nu}$ is canonically normalized. We have also separated the interactions with the gauge fields and the Higgs $T_{B}^{\mu \nu}$ from the stress energy tensor of fermions $T_{F}^{\mu \nu}$. Other separations could be possible, but for simplicity we use this distinction.

In the limit $m_{\rho} \gg m_{\mathrm{SM}}$ the form of the partial widths are dominated practically by counting degrees of freedom, since the leading contributions from mass terms are proportional to $T^{\mu \nu} \sim \eta^{\mu \nu}$ and thus vanish onshell. The partial decays widths are [56]

$$
\Gamma_{\gamma \gamma} \simeq \frac{m_{\rho}^{3}}{80 \pi \Lambda_{G}^{2}}, \quad \Gamma_{g g} \simeq 8 \Gamma_{\gamma \gamma}, \quad \Gamma_{Z Z} \simeq \frac{13}{12} \Gamma_{\gamma \gamma}, \quad \Gamma_{W W} \simeq \frac{13}{6} \Gamma_{\gamma \gamma}, \quad \Gamma_{h h} \simeq \frac{1}{12} \Gamma_{\gamma \gamma},
$$

and

$$
\Gamma_{\ell \ell} \simeq \frac{m_{\rho}^{3}}{160 \pi \Lambda_{F}^{2}}, \quad \Gamma_{q q} \simeq N_{c} \Gamma_{\ell \ell} .
$$

If the ratio of couplings is $\Lambda_{G} / \Lambda_{F} \ll 1$ the dominant production channel could be gluon fusion. Given that the ratios among boson couplings have been fixed, the diphoton branching ratio is

$$
\mathcal{B R}_{\rho \rightarrow \gamma \gamma} \simeq \frac{3}{37}+\mathcal{O}\left(\frac{\Lambda_{G}^{2}}{\Lambda_{F}^{2}}\right) \sim 8 \% .
$$

The total rate should thus be $20-40 \mathrm{fb}$. Due to the sensitivity of dilepton searches, the branching ratios of leptons must be $\lesssim 1 \%$ which justifies the approximation made. The total rate for fixed $m_{\rho}$ then is a function of $\Lambda_{G}$ which can be selected to achieve the correct rate to explain the excess.

\section{Conclusions}

In this paper we considered a possible framework that can explain the excess reported by ATLAS and CMS in the search for diphoton resonances and and explored the consequences. 
Given the challenges imposed by a resonance observed first in its decay to diphoton, a channel with a notoriously small branching ratio, we focused on a pseudoscalar resonance. This is a scenario where we avoid the very strong limits posed by dilepton and diboson searches (see table 3). As discussed, a proposed singlet pseudoscalar couples to SM particles only at the non-renormalizable level to operators that are SM singlets and CP odd. This list is rather constrained and the leading interactions are to tops, gluons, and photons. We emphasized two possible limits of the effective description. Particles of other natures, like a CP even scalar or a spin-2 particle also face numerous constraints.

The first limit, a "natural scenario", could be offered by new physics in which the suppression scale is common for all operators, thus establishing the coupling of the singlet to the SM top as the leading interaction. In this limit we showed that searches for $t \bar{t}$ resonances from $8 \mathrm{TeV}$ data already constrain part of the parameter space. Moreover, in this case to match the diphoton rate, the coupling to photons requires a sizable contribution from new physics. This case seems difficult to realize in composite Higgs models without adding new states in addition to those from the composite sector. As we have stressed, the challenge is to get a sufficiently large branching ratio to diphoton which could be done by relying on anomalous couplings which are allowed if the global symmetries of the composite sector are anomalous.

The second limit is to assume that the new physics responsible for the effective operators only produces a sizable coupling to the field strengths of the gauge bosons (and not to the fermions). In this case the $t \bar{t}$ constraint is avoided and the excess can be reproduced by invoking sizable effects in $G_{\mu \nu}^{a} \tilde{G}^{\mu \nu a}$. Then the suppression scale of $\sim 3 \mathrm{TeV}$ for the fermion operator (and $\mathcal{O}(1)$ coupling) could be sufficient to explain the excess. Since only moderate values of $c_{\gamma}$ and $c_{g}$ are required, fewer new states are needed relative to the previous case. One drawback is that even if this scenario was realized in the composite picture, one still requires a moderate tuning of the size of the coupling between the singlet and the top.

A common aspect to both viable scenarios is that the diphoton excess can only be explained if a sector of new particles and interactions is present at a relative low scale, comparable or possibly even lower than $750 \mathrm{GeV}$. In particular the presence of colored and electroweakly charged states seems unavoidable. Moreover, in the case of composite models with anomalous contributions to the decay channels, one expects colored (and possibly long lived) pions that might be accessible at LHC. The diphoton excess represents an exciting prospect as Run 2 has only just started. Forthcoming data will tell us more.

\section{Acknowledgments}

The authors would like to thank Nima Arkani-Hamed, Gustavo Burdman, Raffaele Tito D'Agnolo, and David Pinner for useful conversations. AT thanks Marco Farina and Michele Redi for interesting comments. ML is supported by a Peter Svennilson Membership at the Institute for Advanced Study, AT is supported by an Oehme Fellowship, and LTW is supported by DOE grant DE-SC0013642. 


\section{A $\quad \mathrm{SO}(6) / \mathrm{SO}(5)$ model}

In this appendix we derive some useful formulas for the $\mathrm{SO}(6) / \mathrm{SO}(5)$ composite Higgs model with a Higgs and a pseudoscalar $\eta$. This section is intended to clarify some of the estimates and arguments given in section 4 with the aid of an explicit case.

\section{A.1 The gauge sector}

The standard model $\mathrm{SU}(2)_{L} \times \mathrm{U}(1)_{Y}$ is embedded in an $\mathrm{SO}(4)$ subgroup of the unbroken $\mathrm{SO}(5)$, under which the $\eta$ is an exact goldstone. The "pions" of the coset space can be organized in the matrix

$$
\Pi=\sqrt{2}\left(h^{i} T^{i}+\eta T_{\eta}\right)=\left(\begin{array}{cc|c}
\mathbf{0}_{4} & 0 & \vec{h} \\
0 & 0 & \eta \\
\hline-\vec{h}^{T} & -\eta & 0
\end{array}\right),
$$

where $T^{i}(i=1,2,3,4)$ and $T_{\eta}$ are the broken generators of $\mathrm{SO}(6)$. For convention we gauge the $\mathrm{SU}(2)_{L} \times \mathrm{U}(1)_{Y}$ subgroup in the upper left $4 \times 4$ block, which is consistent with the assumption of $\eta$ being a SM singlet. We then define the vector $\Sigma_{i} \equiv[\exp (i \Pi / f)]_{i}{ }^{6}$ as (where now $i=1, \ldots, 6$ )

$$
\Sigma^{T}=\frac{\sin \left(\frac{\sqrt{h^{2}+\eta^{2}}}{f}\right)}{\frac{\sqrt{h^{2}+\eta^{2}}}{f}}\left(\vec{h}, \eta, f \cot \left(\frac{\sqrt{h^{2}+\eta^{2}}}{f}\right)\right),
$$

where $h^{2}=\vec{h}^{2}$. We perform a field redefinition [43]

$$
\vec{h} \leftarrow f \frac{\vec{h}}{\sqrt{h^{2}+\eta^{2}}} s, \quad \eta \leftarrow f \frac{\eta}{\sqrt{h^{2}+\eta^{2}}} s,
$$

where $s=\sin \left(\frac{\sqrt{h^{2}+\eta^{2}}}{f}\right)$. In terms of the new fields, the goldstone multiplet is

$$
\Sigma^{T}=\left(\vec{h}, \eta, \sqrt{f^{2}-h^{2}-\eta^{2}}\right),
$$

which leads to the following effective lagrangian (in unitary gauge)

$$
\begin{aligned}
\mathcal{L} & =\frac{1}{2}\left(D^{\mu} \Sigma\right)^{T} D_{\mu} \Sigma \\
& =\frac{1}{2}\left(\partial_{\mu} h\right)^{2}+\frac{1}{2}\left(\partial_{\mu} \eta\right)^{2}+\frac{1}{2 f^{2}}\left(h \partial_{\mu} h+\eta \partial_{\mu} \eta\right)^{2}+\frac{g^{2}}{4} h^{2} W_{\mu}^{a} W^{\mu a}+\ldots
\end{aligned}
$$

In this basis it is manifest that $\langle h\rangle=v=246 \mathrm{GeV}$ and the $\eta$ does not contribute to the electroweak vacuum expectation value. From eq. (A.5), however, we see that after electroweak symmetry breaking there will be a non canonically normalized kinetic term for $h$. The following shift

$$
h \rightarrow v+\sqrt{1-\frac{v^{2}}{f^{2}}} h, \quad \eta \rightarrow \eta,
$$


restores canonical normalization and allows us to compute the couplings to vectors

$$
\frac{g_{h V V}}{g_{h V V}^{\mathrm{SM}}}=\sqrt{1-\frac{v^{2}}{f^{2}}}, \quad \frac{g_{\eta V V}}{g_{h V V}^{\mathrm{SM}}}=0,
$$

where $g_{h V V}^{\mathrm{SM}}$ is the SM coupling.

Anomalies. The global symmetry $\mathrm{SO}(6) \simeq \mathrm{SU}(4)$ can have anomalies. In terms of the $\mathrm{SU}(4)$ generators the embedding of the $\mathrm{SM}$ is

$$
T_{L}^{a} \sim\left(\begin{array}{c}
\sigma^{a} \\
\end{array}\right), \quad T_{R}^{a} \sim\left(\begin{array}{c} 
\\
\sigma^{a}
\end{array}\right), \quad T_{\eta} \sim\left(\begin{array}{cc}
\mathbb{I} & \\
& -\mathbb{I}
\end{array}\right),
$$

while $\mathrm{U}(1)_{X}$ is an external abelian factor. Global anomalies of $\mathrm{SU}(4)^{3}$ induce anomalous couplings of the $\eta$ to SM gauge fields, with the anomaly coefficients of $\mathrm{SU}(2)_{L}, c_{W}$, and hypercharge, $c_{B}$, fixed by the embedding of the SM inside SU(4) to satisfy $c_{W}+c_{B}=0$, as can be explicitly checked. Indeed, the generator of the singlet is $T_{\eta} \sim \operatorname{diag}(1,1,-1,-1)$ while $T_{\mathrm{em}} \sim \operatorname{diag}(1,-1,1,-1)+q_{X} \mathbb{I}$, where $q_{X}$ is a charge of an additional $U(1)_{X}$.

\section{A.2 The fermion sector}

As discussed in eq. (4.4) the SM fermions are embedded in incomplete representations of $\mathrm{SO}(6)$. More precisely the global group needs to be $\mathrm{SO}(6) \times \mathrm{U}(1)_{X}$ where $\mathrm{SM}$ hypercharge is defined as $Y=X+T_{R}^{3}$. Among the several irreducible representations of $\mathrm{SO}(6)$, we consider here the $\boldsymbol{6}_{2 / 3}$ which decomposes under $\mathrm{SO}(5) \times \mathrm{U}(1)_{X}$ as a $\mathbf{5}_{2 / 3}+\mathbf{1}_{2 / 3}+\mathbf{1}_{2 / 3}$. Under the $\mathrm{SU}(2)_{L} \times \mathrm{SU}(2)_{R}$ the decomposition is

$$
\mathbf{6}_{2 / 3} \rightarrow(\mathbf{2}, \mathbf{2})_{2 / 3}+(\mathbf{1}, \mathbf{1})_{2 / 3}+(\mathbf{1}, \mathbf{1})_{2 / 3} .
$$

We can embed quark doublet $q_{L}$ in the bidoublet component, while the $u_{R}$ can be embedded in a linear combination of the two singlets. An embedding that is consistent with our assumption of CP conservation, also at the level of the composite sector, is

$$
q_{L}^{T}=\frac{1}{\sqrt{2}}\left(i b_{L}, b_{L}, i t_{L},-t_{L}, 0,0\right), \quad u_{R}^{T}=(0,0,0,0, i \cos \theta, \sin \theta) u_{R} .
$$

Eq. (A.10) shows that the mixing of $q_{L}$ does not break the shift symmetry of $\eta$ (i.e. $T_{\eta} q_{L}=0$ ) while in general the mixing of $u_{R}$ does break it. Depending on value of $\theta$, which controls the coupling of $u_{R}$ to $\eta$, one can have different scenarios.

For $\theta=\pi / 4, \eta$ is an exact goldstone since the mixing respects the $\mathrm{U}(1)_{\eta}$ symmetry that is generated by $T_{\eta}$. Even though it is a goldstone it still couples to $u_{R}$. On the other hand, for $\theta=\pi / 2$, the mixing respects a discrete $Z_{2}$ symmetry, but $\eta$ does not couple to fermions. In the discussion in section 4 we implicitly avoided these two limiting cases to ensure a coupling between the $\eta$ and $t \bar{t}$. Lighter quarks, however, can have different embeddings and one can even choose embeddings to automatically satsify $\theta=\pi / 2$ or $\theta=\pi / 4$ [57].

The form of the Yukawa term is constrained to be

$$
y_{t}\left(q_{L} \Sigma\right)\left(\Sigma^{T} u_{R}\right)=\frac{y_{t}}{\sqrt{2}} \bar{t}_{L} h t_{R}\left(\frac{\sqrt{f^{2}-h^{2}-\eta^{2}}}{f^{2}} \sin \theta+\frac{\eta}{f} i \cos \theta\right)+\text { h.c. }
$$


Normalizing to the SM Higgs couplings we have the following couplings to fermions for $h$ and $\eta$

$$
\frac{g_{h t t}}{g_{h t t}^{\mathrm{SM}}}=\frac{1-\frac{2 v^{2}}{f^{2}}}{\sqrt{1-\frac{v^{2}}{f^{2}}}}, \quad \frac{g_{\eta t t}}{g_{h t t}^{\mathrm{SM}}}=i \frac{v}{f} \frac{\cot \theta}{\sqrt{1-\frac{v^{2}}{f^{2}}}},
$$

to be compared with eq. (4.6). Notice that since the top mass is proportional to $\sin \theta$ smaller values of $\sin \theta$ will increase the coupling of the top to the pseudoscalar, but will also induce tuning among the parameters of the model.

Other terms can be written with the $\Sigma$, an example used in section 4 are chirality preserving operators that can induce a leading contribution to the potential for $\eta$, as

$$
\overline{u_{R}} \not p \Sigma^{T} \Sigma u_{R}=\overline{u_{R}} \not p u_{R}\left(\eta^{2} \cos ^{2} \theta+\left(f^{2}-h^{2}-\eta^{2}\right) \sin ^{2} \theta\right),
$$

which justifies the expression in eq. (4.8).

Contributions to $c_{\gamma}$ and $c_{g}$ from top partners. A refined estimate for the UV contribution to $c_{\gamma}$ and $c_{g}$ from the top partners involves the full mass spectrum of the heavy fermions. In order to be explicit, we consider the case where the left handed and right handed elementary quarks each couple to a $\mathbf{6}$ of SO(6). The $\mathbf{6}$ decomposes as a $\mathbf{5}+$ $\mathbf{1}$, the states for which we denote as $\Psi_{5}$ and $\Psi_{1}$, respectively. They lead to the mass terms

$$
\mathcal{L} \supset y_{L} f \overline{q_{L}} U \Psi_{R}+y_{R} f \overline{\Psi_{L}} U u_{R}-m_{5} \bar{\Psi}_{5 L} \Psi_{5 R}-m_{1} \bar{\Psi}_{1 L} \Psi_{1 R}+\text { h.c. }
$$

The states of the $\mathbf{6}$ are

$$
\Psi=\frac{1}{\sqrt{2}}\left(\begin{array}{c}
i B-i X_{5 / 3} \\
B+X_{5 / 3} \\
i X_{2 / 3}+i T \\
X_{2 / 3}-T \\
i \sqrt{2} T_{a} \\
\sqrt{2} T_{b}
\end{array}\right)
$$

where under the SM these are $(T, B),\left(X_{5 / 3}, X_{2 / 3}\right), T_{a}$, and $T_{b}$ which are respectively a $\mathbf{2}_{7 / 6}$, a $\mathbf{2}_{1 / 6}$, a $\mathbf{1}_{2 / 3}$, and a $\mathbf{1}_{2 / 3}$. The upper 5 components comprise $\Psi_{5}$ and the lowest is $\Psi_{1}$.

The actual calculation of the effective coupling to the field strengths can be simplified using the Higgs low energy theorem that allows us to compute the contribution using only the mass spectrum. In particular, for a top partner $\Psi^{i}$ we need to know the Yukawa coupling $g_{i}$ and the mass $m_{i}$ defined as $i g_{i} \eta \Psi^{i} \gamma_{5} \Psi^{i}$ and $m_{i} \Psi^{i} \Psi^{i}$. With reference to eq. (A.14) we note that $m_{i}$ is a function of $h$ and $\eta$, but given the assumption of CP conservation $m_{i}=m_{i}(\eta=0)$. On the other hand, $g_{i}$ can be computed from the imaginary part of the mass matrix $\mathcal{M}$ in the background of $\eta, i g_{i}=\partial m_{i} /\left.\partial \eta\right|_{\eta=0}$. The following relation holds,

$$
\sum_{i} \frac{g_{i}}{m_{i}}=\frac{\partial}{\partial \eta} \log \operatorname{det} \mathcal{M}=\frac{1}{f} \frac{\cot \theta}{\sqrt{1-v^{2} / f^{2}}}
$$

where $i$ runs over the fermion states including the SM top. This contribution is equal to the contribution just from the top in eq. (A.12) which means that the contribution of 
fermions much heavier than $\eta$ vanishes. Notice that differently from the case of the Higgs couplings [58] here the wave function renormalization of the light quarks does not introduce new effects (unless CP is broken).

The overall contribution from top partners is then

$$
\frac{c_{\gamma}}{\Lambda}=\frac{4}{3} \sum_{i} \frac{g_{i}}{m_{i}} A_{-}\left(\tau_{i}\right) \simeq \frac{1}{f} \cdot \mathcal{O}\left(\frac{m_{t}}{m_{*}} \frac{m_{\eta}^{2}}{m_{*}^{2}}\right)
$$

This suggests that one has to deviate from the limit of all heavy top partners, however, as discussed in section 4 , it seems challenging to achieve the size needed for $c_{\gamma}$ and $c_{g}$ solely from top partners and comply with the direct limits on their masses.

Open Access. This article is distributed under the terms of the Creative Commons Attribution License (CC-BY 4.0), which permits any use, distribution and reproduction in any medium, provided the original author(s) and source are credited.

\section{References}

[1] ATLAS collaboration, Search for resonances decaying to photon pairs in $3.2 b^{-1}$ of $p p$ collisions at $\sqrt{s}=13$ TeV with the ATLAS detector, ATLAS-CONF-2015-081 (2015).

[2] CMS collaboration, Search for new physics in high mass diphoton events in proton-proton collisions at $\sqrt{s}=13$ TeV, CMS-PAS-EXO-15-004 (2015).

[3] D. Buttazzo, A. Greljo and D. Marzocca, Knocking on new physics' door with a scalar resonance, Eur. Phys. J. C 76 (2016) 116 [arXiv:1512.04929] [INSPIRE].

[4] R. Franceschini et al., What is the gamma gamma resonance at 750 GeV?, arXiv: 1512.04933 [INSPIRE].

[5] S. Di Chiara, L. Marzola and M. Raidal, First interpretation of the 750 GeV di-photon resonance at the $L H C$, arXiv:1512.04939 [INSPIRE].

[6] Y. Mambrini, G. Arcadi and A. Djouadi, The LHC diphoton resonance and dark matter, Phys. Lett. B 755 (2016) 426 [arXiv:1512.04913] [INSPIRE].

[7] M. Backovic, A. Mariotti and D. Redigolo, Di-photon excess illuminates dark matter, arXiv: 1512.04917 [INSPIRE].

[8] A. Angelescu, A. Djouadi and G. Moreau, Scenarii for interpretations of the LHC diphoton excess: two Higgs doublets and vector-like quarks and leptons, arXiv:1512.04921 [INSPIRE].

[9] S. Knapen, T. Melia, M. Papucci and K. Zurek, Rays of light from the LHC, arXiv: 1512.04928 [INSPIRE].

[10] K. Harigaya and Y. Nomura, Composite Models for the 750 GeV diphoton excess, Phys. Lett. B 754 (2016) 151 [arXiv: 1512.04850] [INSPIRE].

[11] Y. Nakai, R. Sato and K. Tobioka, Footprints of new strong dynamics via anomaly, arXiv: 1512.04924 [INSPIRE].

[12] A. Pilaftsis, Diphoton signatures from heavy axion decays at the CERN Large Hadron Collider, Phys. Rev. D 93 (2016) 015017 [arXiv:1512.04931] [InSPIRE]. 
[13] ATLAS collaboration, Search for high-mass diphoton resonances in pp collisions at $\sqrt{s}=8$ TeV with the ATLAS detector, Phys. Rev. D 92 (2015) 032004 [arXiv:1504.05511] [INSPIRE].

[14] CMS collaboration, Search for high-mass diphoton resonances in pp collisions at $\sqrt{s}=8 \mathrm{TeV}$ with the CMS detector, CMS-PAS-EXO-12-045 (2012).

[15] CMS collaboration, Search for diphoton resonances in the mass range from 150 to $850 \mathrm{GeV}$ in pp collisions at $\sqrt{s}=8 \mathrm{TeV}$, Phys. Lett. B 750 (2015) 494 [arXiv:1506.02301] [InSPIRE].

[16] ATLAS and CMS collaborations, ATLAS and CMS physics results from Run 2, December 15, CERN, Switzerland (2015), see webpage.

[17] ATLAS collaboration, A search for $t \bar{t}$ resonances using lepton-plus-jets events in proton-proton collisions at $\sqrt{s}=8$ TeV with the ATLAS detector, JHEP 08 (2015) 148 [arXiv: 1505.07018] [INSPIRE].

[18] CMS collaboration, Search for resonant t $\bar{t}$ production in proton-proton collisions at $\sqrt{s}=8$ TeV, Phys. Rev. D 93 (2016) 012001 [arXiv:1506.03062] [InSPIRE].

[19] CMS collaboration, Search for neutral MSSM Higgs bosons decaying into a pair of bottom quarks, JHEP 11 (2015) 071 [arXiv:1506.08329] [INSPIRE].

[20] ATLAS collaboration, Search for new resonances in $W \gamma$ and $Z \gamma$ final states in pp collisions at $\sqrt{s}=8 \mathrm{TeV}$ with the ATLAS detector, Phys. Lett. B 738 (2014) 428 [arXiv:1407.8150] [INSPIRE].

[21] ATLAS collaboration, Search for an additional, heavy Higgs boson in the $H \rightarrow Z Z$ decay channel at $\sqrt{s}=8 \mathrm{TeV}$ in pp collision data with the ATLAS detector, Eur. Phys. J. C 76 (2016) 45 [arXiv: 1507.05930] [INSPIRE].

[22] ATLAS collaboration, Search for resonant diboson production in the $\ell \ell q \bar{q}$ final state in $p p$ collisions at $\sqrt{s}=8 \mathrm{TeV}$ with the ATLAS detector, Eur. Phys. J. C 75 (2015) 69 [arXiv: 1409.6190] [INSPIRE].

[23] CMS collaboration, Search for a Higgs boson in the mass range from 145 to $1000 \mathrm{GeV}$ decaying to a pair of $W$ or $Z$ bosons, JHEP 10 (2015) 144 [arXiv:1504.00936] [INSPIRE].

[24] CMS collaboration, Search for massive resonances decaying into pairs of boosted bosons in semi-leptonic final states at $\sqrt{s}=8 \mathrm{TeV}$, JHEP 08 (2014) 174 [arXiv:1405.3447] [INSPIRE].

[25] ATLAS collaboration, Search for production of $W W / W Z$ resonances decaying to a lepton, neutrino and jets in pp collisions at $\sqrt{s}=8 \mathrm{TeV}$ with the ATLAS detector, Eur. Phys. J. C 75 (2015) 209 [Erratum ibid. C 75 (2015) 370] [arXiv: 1503.04677] [INSPIRE].

[26] ATLAS collaboration, Search for new phenomena in the dijet mass distribution using pp collision data at $\sqrt{s}=8 \mathrm{TeV}$ with the ATLAS detector, Phys. Rev. D 91 (2015) 052007 [arXiv:1407.1376] [INSPIRE].

[27] CMS collaboration, Search for resonances decaying to dijet final states at $\sqrt{s}=8 \mathrm{TeV}$ with scouting data, CMS-PAS-EXO-14-005 (2014).

[28] ATLAS collaboration, Search for high-mass dilepton resonances in pp collisions at $\sqrt{s}=8 \mathrm{TeV}$ with the ATLAS detector, Phys. Rev. D 90 (2014) 052005 [arXiv:1405.4123] [INSPIRE].

[29] CMS collaboration, Search for physics beyond the standard model in dilepton mass spectra in proton-proton collisions at $\sqrt{s}=8 \mathrm{TeV}$, JHEP 04 (2015) 025 [arXiv:1412.6302] [INSPIRE]. 
[30] ATLAS collaboration, Search for Higgs boson pair production in the $b \bar{b} b \bar{b}$ final state from $p p$ collisions at $\sqrt{s}=8 \mathrm{TeV}$ with the ATLAS detector, Eur. Phys. J. C 75 (2015) 412 [arXiv: 1506.00285] [INSPIRE].

[31] CMS collaboration, Search for resonant pair production of Higgs bosons decaying to two bottom quark-antiquark pairs in proton-proton collisions at 8 TeV, Phys. Lett. B 749 (2015) 560 [arXiv: 1503.04114] [INSPIRE].

[32] CMS collaboration, Search for neutral MSSM Higgs bosons decaying to a pair of $\tau$ leptons in pp collisions, JHEP 10 (2014) 160 [arXiv:1408.3316] [INSPIRE].

[33] ATLAS collaboration, Search for neutral Higgs bosons of the minimal supersymmetric standard model in pp collisions at $\sqrt{s}=8 \mathrm{TeV}$ with the ATLAS detector, JHEP 11 (2014) 056 [arXiv: 1409.6064] [INSPIRE].

[34] ATLAS collaboration, Search for a CP-odd Higgs boson decaying to Zh in pp collisions at $\sqrt{s}=8 \mathrm{TeV}$ with the ATLAS detector, Phys. Lett. B 744 (2015) 163 [arXiv:1502.04478] [INSPIRE].

[35] CMS collaboration, Search for dark matter, extra dimensions and unparticles in monojet events in proton-proton collisions at $\sqrt{s}=8$ TeV, Eur. Phys. J. C 75 (2015) 235 [arXiv: 1408.3583] [INSPIRE].

[36] ATLAS collaboration, Search for new phenomena in final states with an energetic jet and large missing transverse momentum in pp collisions at $\sqrt{s}=8$ TeV with the ATLAS detector, Eur. Phys. J. C 75 (2015) 299 [arXiv:1502.01518] [INSPIRE].

[37] TWIKI, SM Higgs production cross sections at 13-14 TeV, https://twiki.cern.ch/twiki/bin/view/LHCPhysics/CERNYellowReportPageAt1314TeV (2015).

[38] B.C. Allanach, P.S.B. Dev, S.A. Renner and K. Sakurai, Di-photon excess explained by a resonant sneutrino in R-parity violating supersymmetry, arXiv:1512.07645 [INSPIRE].

[39] M. Spira, HIGLU: a program for the calculation of the total Higgs production cross-section at hadron colliders via gluon fusion including QCD corrections, hep-ph/9510347 [INSPIRE].

[40] LHC Higgs Cross Section Working Group collaboration, J.R. Andersen et al., Handbook of LHC Higgs cross sections: 3. Higgs properties, arXiv:1307.1347 [INSPIRE].

[41] G. Panico and A. Wulzer, The composite Nambu-Goldstone Higgs, Lect. Notes Phys. 913 (2016) 1 [arXiv: 1506.01961] [INSPIRE].

[42] K. Agashe, R. Contino and A. Pomarol, The minimal composite Higgs model, Nucl. Phys. B 719 (2005) 165 [hep-ph/0412089] [INSPIRE].

[43] B. Gripaios, A. Pomarol, F. Riva and J. Serra, Beyond the minimal composite Higgs model, JHEP 04 (2009) 070 [arXiv:0902.1483] [InSPIRE].

[44] J. Serra, Beyond the minimal top partner decay, JHEP 09 (2015) 176 [arXiv:1506.05110] [INSPIRE].

[45] E. Katz, A.E. Nelson and D.G.E. Walker, The intermediate Higgs, JHEP 08 (2005) 074 [hep-ph/0504252] [INSPIRE].

[46] J. Galloway, J.A. Evans, M.A. Luty and R.A. Tacchi, Minimal conformal technicolor and precision electroweak tests, JHEP 10 (2010) 086 [arXiv:1001.1361] [INSPIRE]. 
[47] J. Mrazek, A. Pomarol, R. Rattazzi, M. Redi, J. Serra and A. Wulzer, The other natural two Higgs doublet model, Nucl. Phys. B 853 (2011) 1 [arXiv:1105.5403] [INSPIRE].

[48] ATLAS collaboration, Measurements of the Higgs boson production and decay rates and coupling strengths using pp collision data at $\sqrt{s}=7$ and $8 \mathrm{TeV}$ in the ATLAS experiment, Eur. Phys. J. C 76 (2016) 6 [arXiv:1507.04548].

[49] CMS collaboration, Precise determination of the mass of the Higgs boson and tests of compatibility of its couplings with the standard model predictions using proton collisions at 7 and $8 \mathrm{TeV}$, Eur. Phys. J. C 75 (2015) 212 [arXiv:1412.8662] [INSPIRE].

[50] D.B. Kaplan, Flavor at SSC energies: a new mechanism for dynamically generated fermion masses, Nucl. Phys. B 365 (1991) 259 [INSPIRE].

[51] O. Matsedonskyi, G. Panico and A. Wulzer, On the interpretation of top partners searches, JHEP 12 (2014) 097 [arXiv: 1409.0100] [INSPIRE].

[52] J.A. Aguilar-Saavedra, R. Benbrik, S. Heinemeyer and M. Pérez-Victoria, Handbook of vectorlike quarks: mixing and single production, Phys. Rev. D 88 (2013) 094010 [arXiv: 1306.0572] [INSPIRE].

[53] R. Contino, Y. Nomura and A. Pomarol, Higgs as a holographic pseudo-Goldstone boson, Nucl. Phys. B 671 (2003) 148 [hep-ph/0306259] [INSPIRE].

[54] M. Schmaltz, The simplest little Higgs, JHEP 08 (2004) 056 [hep-ph/0407143] [INSPIRE].

[55] K. Hinterbichler, Theoretical aspects of massive gravity, Rev. Mod. Phys. 84 (2012) 671 [arXiv:1105.3735] [INSPIRE].

[56] T. Han, J.D. Lykken and R.-J. Zhang, On Kaluza-Klein states from large extra dimensions, Phys. Rev. D 59 (1999) 105006 [hep-ph/9811350] [InSPIRE].

[57] M. Frigerio, A. Pomarol, F. Riva and A. Urbano, Composite scalar dark matter, JHEP 07 (2012) 015 [arXiv: 1204.2808] [INSPIRE].

[58] A. Azatov and J. Galloway, Light custodians and Higgs physics in composite models, Phys. Rev. D 85 (2012) 055013 [arXiv:1110.5646] [INSPIRE]. 